\title{
Marketing Digital como oportunidad de digitalización de las PYMES en Colombia en tiempo del Covid - 19
}

\section{Digital Marketing as a digitalization opportunity for SMEs in Colombia in the time of Covid - 19}

Sherly Hoyos-Estrada

Universidad Tecnológica de Bolívar - Colombia

ORCID iD: https://orcid.org/0000-0003-2533-5344

sherhe@hotmail.com

\section{Judith Daniela Sastoque-Gómez*}

Servicio Nacional de Aprendizaje SENA - Colombia ORCID iD: https://orcid.org/0000-0003-4123-130X jsastoqueg@sena.edu.co

* Autor a quien debe ser dirigida la correspondencia
Fecha de recepción: 15/03/2020 Fecha de evaluación: 28/03/2020

Fecha de aceptación: 10/06/2020

Cómo citar: Hoyos-Estrada, S., \& Sastoque-Gómez, J. (2020). Marketing Digital como oportunidad de digitalización de las PYMES en Colombia en tiempo del Covid - 19. Revista Cientifica Anfibios, 3(1), 39-46. https://doi.org/10.37979/afb.2020v3n1.60

\section{Resumen}

La pandemia del Covid - 19, ha desestabilizado el sistema socioeconómico del país, principalmente afectado a las Pymes, siendo $28 \%$ del PIB, el $67 \%$ del empleo y el $37 \%$ de la producción nacional. El marketing digital se ha convertido en una herramienta de apoyo para las empresas, gracias a esto son más competitiva en el mercado siendo posible por la internet. Actualmente está en riesgo de cierre un $20 \%$ de las pymes del país, lo que significaría perder medio millón de unidades productivas, es por esto que se expone los beneficios del uso de estrategias de marketing digital para la exploración de los mercados digitales, con el fin de salvaguardar la economía nacional, durante y después de la pandemia.

\section{Palabras claves:}

Pymes; Marketing Digital; Estrategias; Covid - 19; Digitalización

\begin{abstract}
The Covid - 19 pandemic has destabilized the country's socioeconomic system, mainly affected by SMEs, with $28 \%$ of GDP, $67 \%$ of employment and $37 \%$ of national production. Digital marketing has become a support tool for companies, thanks to this they are more competitive in the market, being possible through the internet. Currently, $20 \%$ of the country's SMEs are at risk of closure, which would mean losing half a million productive units, which is why the benefits of using digital marketing strategies for exploring digital markets are exposed, with the in order to safeguard the national economy, during and after the pandemic.
\end{abstract}

Keywords:

SMEs; Digital Marketing; Strategies; Covid - 19; Digitalization 


\section{Introducción}

De acuerdo a Castaño \& Jurado (2016), la llegada de lo online generó un cambio en la forma en que nos comunicamos y relacionamos. El marketing Digital ha surgido como una solución a las nuevas tecnologías y una nueva forma de utilizar y aprovechar internet.

Por ejemplo, países como Argentina, sólo en el primer semestre del año 2017 la inversión de publicidad en Internet alcanzó casi la totalidad del año 2016 llegando a los \$1.041 millones entre enero y julio (Consultora adCuality, 2017), lo que he gran medida nos brida una visión de camino a seguir a esta nueva era de la digitalización de los negocios.

La pandemia del Covid - 19, no solo ha sido un desafío para la salud pública, sino adicionalmente para el sistema socioeconómico del mundo. Colombia no se libró de los estragos que causó y sigue causando esta pandemia, desestabilizando la economía y la productividad del país.

El 28\% del PIB, depende de las Pymes (Economía aplicada, 2019), por lo tanto, es importante que estas tengan presencia en internet y sigan siendo vigentes y sobrevivir en esta crisis.

Sin embargo, esta crisis ha permitido acelerar un crecimiento en relación al comercio en línea, el cual es aprovechado por aquellos negocios que cuentan con una fuerte presencia en internet y han logrado una buena reputación online. El Marketing Digital se ha convertido en una herramienta efectiva para las grandes, medianas y pequeñas empresas, con el fin de obtener crecimiento en mercado globalizado. En Colombia y en el mundo, la manera de hacer compras toma otro tono, camino a una era digital, de este modo las empresas reconocen a los dispositivos móviles como un medio de crear vínculos con clientes potenciales.

Para este fin, en este artículo se expondrá la situación de las Pymes en Colombia en medio de la coyuntura y como el Marketing puede ser un beneficio para el comercio tanto físico como digital.

\section{Referentes Teóricos}

Para desarrollar el objetivo principal del presente artículo se centra en examinar algunas herramientas que ofrece el Marketing digital para llevar al comercio electrónico a las Pymes del país durante y post periodo de pandemia, es necesario referenciar algunos conceptos teóricos respecto a estas temáticas.

\section{Importancia de las PYMES en el desarrollo económico del país}

Para este apartado, es oportuno conceptualizar a las pequeñas y medianas empresas (En adelante, PYMES). De acuerdo a la Ley 590 de 2000 y su modificación con la Ley 905 de 2004 (Ley 905 De 2004, 2019), son clasificada de acuerdo al número de empleados, comprendidos entre 1 a 201 y activos especificados en SMLMV (Salario mínimo mensual legal $2020=\$ 877,803)$ entre 501 a 30.001. (Francisco et al., 2020)

De acuerdo a Tabares (2012), la competitividad de un país está vinculado a la competitividad de sus empresas. Como lo menciona Valdaliso \& Lópes (2000), el rol de las PYMES en los países del mundo a la contribución de la tasa de empleo y del PIB, demuestra que son un sector con alta participación en la economía nacional. Las PYMES tienen un papel fundamental e importante en Colombia, es la principalmente por la generación de empleo y su contribución al PIB nacional. (Alexandrov Betov, 2020)

Gómez \& Borda (2018), explican que desde 1992 al 2015, las pequeñas empresas registraron una tasa de crecimiento del $118 \%$, lo cual evidencia una clara generación de crecimiento económico, sin discriminar sectores, proporcionando el 28\% del PIB, el 67\% del empleo y el $37 \%$ de la producción del país. En Colombia hay aproximadamente 109.000 Pymes, equivalen al total de $6,74 \%$ de las empresas registradas en la Cámaras de Comercios regionales (Economía Aplicada, 2019). Cabe mencionar que Colombia cuenta con una alta tasa de comercios no registrados, puede haber cifras oscuras en el cálculo de estos números por su alta tasa de informalidad (Clavijo, 2019). 


\section{Marketing digital}

Es importante remontarse al concepto del comercio electrónico para entender el concepto de marketing digital, este se define como intercambios medidos por la tecnología entre diversas partes (individuos, organizaciones, o ambos), así como las actividades electrónicas dentro y entre organizaciones que faciliten esos intercambios (Rayport, J. F. \& Jaworski, 2001).

El marketing digital ha cambiado y mejorado la manera de hacer negocios, Kotler (2015), afirma que el marketing digital es la combinación de lo tradicional con lo digital. El Instituto Internacional Español de Marketing Digital define el Marketing Digital como un tipo de aplicación de las estrategias de comercialización realizadas a través de los medios digitales (González Bríñez, 2019).
Teniendo en cuenta los conceptos expuesto, cabe mencionar a la web como eje fundamental e implícito del Marketing Digital, debido a que se implementa principalmente por medio buscadores, redes sociales, comercio electrónico, publicidad on-line, elementos de hardware que involucran la tecnología inteligente (IIEMD, n.d.).

\section{Integración del Marketing Digital}

El marketing Digital, es la integración de elementos y herramientas. El Instituto de Mercadotecnia y Publicidad de Guanajuato, México (2015), explica los elementos que forman parte del Marketing Digital y que unen los conceptos mencionados (Ver Tabla. 1). (Medina Acero \& Aguirre Vásquez, 2017)

Tabla 1. Relación de elementos vs desempeño

\begin{tabular}{|c|c|}
\hline Elementos & Desempeño \\
\hline $\begin{array}{l}\text { Dispositivos } \\
\text { (Hardware) }\end{array}$ & $\begin{array}{l}90 \% \text { de las transacciones de los consumidores inician y terminan en un dispositivo, } \\
\text { ese porcentaje en su mayoría son tablets y teléfonos inteligentes }\end{array}$ \\
\hline Búsqueda Orgánica & $\begin{array}{l}\text { Uso de los motores de búsqueda por parte de los clientes, para este punto es } \\
\text { necesario atraerlo y persuadirlo para al negocio. Es necesario, y por mucho, lograr } \\
\text { tener una alta visibilidad y tráfico en la website. }\end{array}$ \\
\hline Content Marketing & $\begin{array}{l}\text { La información proporcionada por la página web y/o redes sociales, debe ser real } \\
\text { y acorde con lo que se ofrece, así será posible garantizar el regreso de los clientes. }\end{array}$ \\
\hline $\begin{array}{l}\text { Social Media } \\
\text { Marketing }\end{array}$ & $\begin{array}{l}\text { En ellas radica el primer paso de contacto con el cliente, se establece una interacción } \\
\text { entre la marca y el usuario. }\end{array}$ \\
\hline E-mail Marketing & $\begin{array}{l}\text { Una correcta campaña de E-Mail permite cerrar un negocio con un cliente } \\
\text { otencial, para esto es necesario contar con un contenido que logré persuadir al } \\
\text { liente. }\end{array}$ \\
\hline $\begin{array}{l}\text { Campañas } \\
\text { publicitarias }\end{array}$ & $\begin{array}{l}\text { medio y el modo de difusión son determinantes para llegar a clientes potenciales, } \\
\text { posible conocer los gustos de los clientes gracias a los datos compartidos por } \\
\text { s usuarios y reacciones del contenido publicado }\end{array}$ \\
\hline Desarrollo web & $\begin{array}{l}\text { esta se debe detallar todo lo que } \\
\text { se muestra el anuncio. }\end{array}$ \\
\hline Imagen corporativa & $\begin{array}{l}\text { Este punto es esencial para el negocio, esta permite establecer una identidad y } \\
\text { reconocimiento entre los usuarios. }\end{array}$ \\
\hline $\begin{array}{l}\text { Posicionamiento en } \\
\text { Google }\end{array}$ & $\begin{array}{l}\text { Un ranking dentro de los motores de búsqueda, no solo dará visibilidad a la marca, } \\
\text { también dará confianza y seguridad. }\end{array}$ \\
\hline $\begin{array}{l}\text { SEO (Search Engine } \\
\text { Optimization) }\end{array}$ & $\begin{array}{l}\text { El fin de mejorar la posición de un website en los resultados de los buscadores } \\
\text { para unos términos de búsqueda concretos que se relacionan directamente con el } \\
\text { negocio. Cabe resaltar que es por medio de crecimiento orgánico. }\end{array}$ \\
\hline $\begin{array}{l}\text { SEM (Search Engine } \\
\text { Marketing) }\end{array}$ & $\begin{array}{l}\text { A diferencia del SEO, este es por publicidad paga. Permite mostrar el sitio cuando } \\
\text { el usuario hace búsquedas con una necesidad en específico y se muestra de primero. }\end{array}$ \\
\hline
\end{tabular}

Fuente: Elaboración propia a partir de datos obtenidos en Instituto de Mercadotecnia

$$
\text { y Publicidad de Guanajuato, México. }
$$


El marketing digital se ha convertido en una herramienta de apoyo para las empresas, gracias a esto son más competitiva en el mercado siendo posible a la internet, quien juega un papel fundamental por su competitividad a nivel mundial.

\section{Metodología}

El presente artículo de revisión se realizó a partir de la investigación de fuentes bibliográficas con referentes nacionales e internacionales. Bajo un modelo de revisión documental, evaluados a partir de su contribución para dar respuesta al interrogante planteado para el desarrollo del presente escrito. ¿Es el Marketing Digital la oportunidad de digitalización de las PYMES en Colombia en tiempo del Covid - 19?

Posterior al análisis crítico de las fuentes consultadas se logró desarrollar una respuesta al interrogante propiamente dicho enunciados en el siguiente apartado.

\section{Resultados}

En primera instancia se exponen la situación de las Pymes en Colombia a partir del Covid - 19, además de realizar un énfasis en las políticas del gobierno para el apoyo de los empresarios y, por ultimo explicar cómo las estrategias de Marketing Digital puede ser un aliado y una oportunidad de crecimiento para las Pymes durante el tiempo de la pandemia y en gran medida posterior a esta.

\section{Situación de las Pymes en Colombia a partir del Covid - 19}

Las Pymes son un factor esencial en la economía del país, proporcionando el $28 \%$ del PIB, el $67 \%$ del empleo y el $37 \%$ de la producción del país. En Colombia hay aproximadamente 109.000 Pymes, equivalen al total de $6,74 \%$ de las empresas registradas en la Cámaras de Comercios regionales (Economía Aplicada, 2019).

A partir del aviso de la cuarentena obligatoria declarada en todo el país desde el 24 de marzo del presente año, los comercios se vieron principalmente afectados debido al confinamiento.

De acuerdo a Serna-Gómez et al., (2020), con el teletrabajo las áreas inmobiliarias y servicios financieros presentan una posibilidad superior al 50\% de capacidad de adaptación para continuar realizando sus actividades económicas, pero para el caso de hoteles y restaurantes, comunicaciones, transporte, comercio, e industria son los principalmente más afectados. Cárdenas \& Montana (2020) explica para el caso de las pymes que participan dentro de las actividades en mención, se verán principalmente afectadas en su tasa de empleabilidad con la pérdida de un $25,7 \%$, lo que equivale a 1.869 .602 personas.

En el estudio realizado Serna-Gómez et al., (2020), cerca del 50\% de los empleados en las Micro y pequeñas empresas, son trabajadores dueños del establecimiento. Las causas de esta pérdida de empleo, son el reflejo del cierre de un gran porcentaje de Pymes, o en mejor de los casos un corte de personal, aproximadamente. En un artículo del Portafolio (2020), explica que de acuerdo a una encuesta "express" realizada por FENALCO, el $90.5 \%$ de los empresarios manifestaron que sus ventas se han visto reducidas, $y$ el $72.1 \%$ calificó tal reducción como alarmante. Asociación Colombiana de Micro, Pequeñas y Medianas empresas (ACOPI), manifiesta el cierre de aproximadamente un $20 \%$ de las empresas en Bogotá y Cundinamarca, lo que equivale a una pérdida de medio millón de unidades productivas del país debido a que el $90 \%$ del comercio del país se concentra en esas zonas. Sin embargo, el gobierno está generando un apoyo económico, tomado la decisión de asumir las nóminas de las pymes por tres meses (Rodríguez Pinzón, 2020).

\section{Marketing digital para el fortalecimiento de las Pymes en Colombia}

En el estudio realizado por Serna-Gómez et al., (2020), identifico que, de las micro y pequeñas empresas del país, el 26,3\% de éstos tienen acceso a internet, el 5,7\% tiene redes sociales, el 7,4\% realiza comercio electrónico y el 10,9\% compra de proveeduría. Se evidencia una cifra alarmante para que estos comercios den el salto hacía la digitalización de sus negocios. 
Moschini (2012) explica que el mercado es dinámico, por lo tanto, mejorar es natural. Conocer las herramientas no basta; y es por ello es necesario entender la interacción con los usuarios. Para Kotler y Armstrong (2017), afirman que el marketing digital y las redes sociales requieren usar recursos tales como: sitios web, anuncios, publicaciones móviles y plataformas digitales que permitan captar la atención de los consumidores, teniendo presente su ubicación geográfica (Juárez et al., 2020).

Los medios digitales están influyendo y generando cambios significativos en las personas, incidiendo además en su transformación cultural, así como la manera en que esas personas se relacionan con las diferentes, marcas producto y servicios (Gallero, 2018) Para ser parte de las Pymes influyentes, es importante contar con una buena reputación online, debido a que es un activo intangible de la empresa relacionado a la percepción que tiene de ella el público y los grupos de interés con los que se relaciona (Flores Torres \& Galarza Uzcategui, 2014).

De acuerdo a Pedraza, Cantillo \& Dueñas (2019), el diseño de estrategias y métodos de marketing no solo permite mantener vigentes a las organizaciones, también permite alcanzar el éxito para el cumplimiento de sus objetivos. Según Thompson \& Strickland (2012), y Durán, García, Parra y García (2018), consideran que las estrategias son un impulso competitivo y un factor importante para las decisiones de los gerentes de relación a los recursos de la compañía para satisfacer a los clientes. Hill \& Jones (2009), afirman que las estrategias son la gestión que el grupo gerencial realiza para lograr cumplir sus metas de la empresa.

La digitalización es un movimiento importante para las Pymes del país, es una estrategia que permite la internacionalización y la supervivencia del negocio de aislamiento que estamos viviendo. La transformación digital y de las diversas estrategias para ofrecer más servicios y productos digitales y físicos, desde algunos años se considera sumamente importante para poder incursionar y sobrevivir en mercados internacionales, siendo las compañías en el área digital las que más dinámicas son y que más rápidamente crecen (Panandiker et al. 2018: 10; MGI 2017: 11).
De acuerdo a Panandiker et al. (2018), la digitalización se debería utilizar para poder penetrar nuevos mercados eficientemente y para adoptar nuevos modelos de negocio digitales, que pueden abrir nuevas oportunidades de negocio.

La Republica (2020) público un estudio en el que afirma que, en América Latina, el aumento de las compras en línea aumento un 300\% durante la pandemia, siendo Colombia quien ocupa el primer lugar de compras en línea. Según estudios de la Cámara Colombiana de Comercio Electrónico (CCE), las ventas en internet han sido una herramienta clave para el abastecimiento de los hogares colombianos, el e-commerce ha crecido entre $50 \%$ y $80 \%$ ante la coyuntura, principalmente en categorías como deportes $(86,5 \%)$, retail $(52,9 \%)$, salud $(38,2 \%)$ y tecnología (26,9\%) (Dinero, 2020).

Esta digitalización puede tener la forma de ofrecer plataformas con la inclusión y aprovechamiento de las herramientas y elementos del Marketing Digital en la cadena de valor, como también otras soluciones dependiendo de la industria (inteligencia artificial, tecnología Blockchain, internet de las cosas, etc.) (MGI 2016: 5; Bianchi, Glavas y Mathews 2016: 188; MGI 2019: 79).

El ROI en una herramienta de medición, el cual es una de las muchas ventajas que ofrece el Marketing Digital para los negocios, permite ajustar la estrategia cuantas veces sea necesario para lograr los objetivos, es más económico que otros métodos, permite realizar una segmentación mucho más personalizada posibilitando generar comunidades de usuarios, etc. (Vercheval, 2016)

A pesar de todas las ventajas que ofrece la transformación digital, una de las desventajas es la falta de contacto directo para crear vínculos entre clientes y empresas fiables (Rintamäki 2017: 99-101). Una cifra alarmante muestra el estudio de McKinsey, estimando entre tres y cinco años de diferencia digital entre las empresas latinas más avanzadas y las empresas norteamericanas de un tamaño similar (MGI 2019: 48), implicando así la necesidad para las empresas latinas de adaptarse a los niveles internacionales para poder 
competir fuera de su mercado local o regional. Poniendo el enfoque sobre las PYMES latinoamericanas, estas tienden a tener mayores problemas en implementar un proceso de digitalización por la falta del acceso al financiamiento, al talento, la infraestructura digital o información sobre prácticas exitosas (MGI 2019: 49), es decir la falta del recurso humano según el Resource-based view (En español - La vista basada en recursos). La falta de financiamiento también se refiere a que las PYMES tradicionalmente son excluidos de las Bolsas como fuente de financiación (Bonnet 2018: 554).

\section{Conclusiones}

Marketing Digital es mercados crecimiento exponencial en las últimas décadas, debido a gran número de empresas apunta a invertir en estas estrategias, reconocen el potencial y alcance a clientes en todo el mundo, no solo productores sino como usuarios de servicios.
El marketing digital son una serie de estrategias que permiten hacer de las Pymes un comercio Internacional, esto es posible con su presencia en las redes sociales y páginas web brindando un mayor alcance de audiencias. No obstante, Colombia necesita fortalecer su conectividad nacional, capacitando a los empresarios con el uso de TIC y normatividad en cuanto a Comercio electrónico además las Pymes, deben diferenciarse con su propuesta de valor en los contenidos, procurando que sea única y relevante, pero también, que no sea estática, porque las necesidades, vínculos, deseos y gustos se modifican con el paso de los años y en los distintos mercados.

Otro factor a tener en cuenta en este artículo es exponer que, si el gobierno brinda incentivos para que los empresarios de la Pymes no solo para tener vigencia de sus negocios físicos, sino adicionalmente presencia digital por lo que la difusión por medios digitales como páginas web, redes sociales, mensajerías de texto o aplicaciones son indispensables y deben tener en espacio dentro de la estructura organizativa de toda empresa que piensa en el posicionamiento de su marca.

\section{Referencias}

Alexandrov Betov, C. (2020). El desarrollo de capacidades para la internacionalización de las PYMES en Colombia. Universidad Salamanca.

Bianchi, C., Glavas, C. y Mathews, S. SME international performance in Latin America: The role of entrepreneurial and technological capabilities. Journal of Small Business and Enterprise Development, 2016, Vol. 24 (1): 176-195.

Bonnet, R. J. Cien Episodios de la Historia Económica de Colombia. Bogotá: Ariel, 2018.

Clavijo, S. (2019). Grados de formalización en las microempresas de Colombia. Revista La República. https:// www.larepublica.co/analisis/sergio-clavijo-500041/grados-de-formalizacion-en-las-microempresasde-colombia-2740128

Dinero. (2020). Comercio electrónico en Colombia, el que más crece de Latinoamérica. https://www.dinero.com/empresas/articulo/comercio-electronico-en-colombia-crece-durante-pandemiapor-coronavirus/286562

Duran, S.; García, J. Parra, M. \& García, M. (2018). Estrategias para disminuir el síndrome de Burnout en personal que labora en Instituciones de salud en Barranquilla. Cultura Educación Y Sociedad, 9 (1), $27-44$

Economía Aplicada. (2019). ¿Cuántas empresas hay en Colombia? http://economiaaplicada.co/index.php/10noticias/1493-2019-cuantas-empresas-hay-en-colombia

Flores Torres, R., \& Galarza Uzcategui, A. (2014). Importancia de la reputacion online para una pyme. http://201.159.223.2/handle/123456789/938 
Francisco, J., Galvis, R., Hernández, Y. G., Andrea, J., \& Campos, C. (2020). Indicadores de gestión como herramienta de diagnóstico para Pymes. I+D Revista de Investigaciones, 15(2256-1676), 109-124.

Gallero, F. (2018). Tendencias del marketing digital desde la perspectiva de las pymes (p. 13). Universidad Siglo 21.

Gómez, H., \& Borda, S. (2018). Diagnóstico de las micro, pequeñas y medianas empresas: políticas e instituciones de fomento en Colombia. In MIPYMES en América Latina Un frágil desempeño y nuevos desafios para las políticas de fomento (pp. 227-228).

González Bríñez, M. H. (2019). El Marketing Digital Transforma La Gestión De Pymes En Colombia. Cuadernos Latinoamericanos de Administración, 14(27). https://doi.org/10.18270/cuaderlam.v14i27.2652

Hill, C y Jones, G. (2009). Administración Estratégica. México: Mc Graw-Hill.

IIEMD. (n.d.). Qué es Marketing Digital? https://iiemd.com/\%0Aque-es-marketing-digital/

Juárez, L., Alejandro, L., Tirado, M., \& López, R. B. (2020). Online marketing : rentabilidad al alcance de las Mipymes. 0104, 57-76.

Kotler, P. E. al. (2015). NoMarketing 4.0. New Jersey: John Wiley \& Sons, Inc., Hoboken.

Kotler, P. y Armstrong, G. (2017). Principios de Marketing. México: Pearson Educación.

La Republica. (2020). Comercio electrónico ha crecido más de 300\% en Latinoamérica en la pandemia. https://www.larepublica.co/globoeconomia/e-commerce-ha-crecido-mas-de-300-en-latinoamerica-enmedio-de-la-pandemia-3000424

Mckinsey Global Institute (MGI). Can Latin America reignite growth by connecting with consumers? McKinsey Global Institute, 2016.

Mckinsey Global Institute (MGI). Where Will Latin America's Growth Come From? McKinsey Global Institute, 2017.

Mckinsey Global Institute (MGI). Latin America's missing middle: Rebooting inclusive growth. McKinsey Global Institute, 2019.

Medina Acero, C. A., \& Aguirre Vásquez, A. (2017). Aplicación para la gestión del cambio del marketing tradicional al Marketing Digital e innovación abierta con énfasis en investigación aplicada para la planeación, para las Pymes y Mipymes en la ciudad de Bogotá. Revista RETO (Colombia), 5(5), 60-73. https://doi.org/4397-1-10-20180412

Moschini, S. (2012). Claves del marketing digital. La nueva comunicación empresarial en el mundo 3.0. Barcelona: Ebooks de Vanguardia.

Panandiker, R. et al. (2018) The 2018 BCG Local Dynamos - Emerging-Market Companies Up Their Game. The Boston Consulting Group, 2018.

Pedraza, C; Cantillo, N \& Dueñas, J (2019). Emprendimiento Social en el sector lácteo. Colombia: Editorial, Universidad de la Guajira.

Portafolio. (2020). Ley de Insolvencia podría salvar empresas en riesgo por Covid-19. https://www.portafolio.co/negocios/empresas/ley-de-insolvencia-podria-salvar-empresas-en-riesgopor-covid-19-539637

Rayport, J. F. \& Jaworski, B. J. (2001). e-Commerce (McGraw Hill. (ed.)).

Rintamäki, P. (2017) Overcoming the Barriers to E-Commerce in Latin America - the Internationalization Perspective. Lappeenranta: Lappeenranta University of Technology. 
Sherly Hoyos-Estrada, Judith Daniela Sastoque-Gómez

Rodríguez Pinzón, É. (2020). Colombia. Impacto económico, social y político de la COVID-19. Análisis Carolina, 1-14. https://doi.org/10.33960/ac 24.2020

Ley 905 De 2004, (2019). http://www.secretariasenado.gov.co/senado/basedoc/ley_0905_2004.html

Serna-Gómez, H. M., Barrera-Escobar, A., \& Castro-Escobar, E. S. (2020). Efectos en el empleo en las micro y pequeñas empresas generados por la emergencia del COVID-19: Caso Colombia. SSRN Electronic Journal. https://doi.org/10.2139/ssrn.3609574

Tabares, A. S. (2012). Revisión analítica de los procesos de Internacionalización de las PYMES (pp. 67-92). Pensamiento y gestión, Universidad del Norte.

Thompson, A y Strickland, A. (2012). Administración Estratégica. México: Mc Graw-Hill.

Valdaliso, J. M., \& Lópes, S. (2000). Historia económica de la empresa. Editorial Crítica. 